\title{
VALIDATION OF MODELLING THE RADIATION EXPOSURE DUE TO SOLAR PARTICLE EVENTS AT AIRCRAFT ALTITUDES
}

\author{
P. Beck ${ }^{1, *}$, D. T. Bartlett ${ }^{2}$, P. Bilski ${ }^{3}$, C. Dyer ${ }^{4}$, E. Flückiger ${ }^{5}$, N. Fuller ${ }^{6}$, P. Lantos ${ }^{6 \dagger}$, G. Reitz $^{7}$, W. Rühm ${ }^{8}$, \\ F. Spurny ${ }^{9}$, G. Taylor ${ }^{10}$, F. Trompier ${ }^{11}$ and F. Wissmann ${ }^{12}$ \\ ${ }^{1}$ Austrian Research Centers, ARC, Radiation Safety and Applications Division, A-2444 Seibersdorf, Austria \\ ${ }^{2}$ Health Protection Agency, London, UK \\ ${ }^{3}$ Institute of Nuclear Physics, Krakow, Poland \\ ${ }^{4}$ QinetiQ, Space Division, Dartford, UK \\ ${ }^{5}$ University of Bern, Bern, Switzerland \\ ${ }^{6}$ Paris-Meudon Observatoire, Meudon, France \\ ${ }^{7}$ Radiation Biology Department, German Aerospace Center, Cologne, Germany \\ ${ }^{8}$ Helmholzentrum München, Institute of Radiation Protection, Neuherberg, Germany \\ ${ }^{9}$ Czech Academy of Science, Department of Radiation Dosimetry, Nuclear Physics Institute, Prague, Czech \\ Republic \\ ${ }^{10}$ National Physical Laboratory, Hampton Road, Teddington, Middlesex, TW11 0LW, UK \\ ${ }^{11}$ Institute de Radioprotection et de Sûreté Nucléaire, Villejuif, France \\ ${ }^{12}$ Physikalisch-Technische Bundesanstalt, D-38116 Braunschweig, Germany
}

\begin{abstract}
Dose assessment procedures for cosmic radiation exposure of aircraft crew have been introduced in most European countries in accordance with the corresponding European directive and national regulations. However, the radiation exposure due to solar particle events is still a matter of scientific research. Here we describe the European research project CONRAD, WP6, Subgroup-B, about the current status of available solar storm measurements and existing models for dose estimation at flight altitudes during solar particle events leading to ground level enhancement (GLE). Three models for the numerical dose estimation during GLEs are discussed. Some of the models agree with limited experimental data reasonably well. Analysis of GLEs during geomagnetically disturbed conditions is still complex and time consuming. Currently available solar particle event models can disagree with each other by an order of magnitude. Further research and verification by on-board measurements is still needed.
\end{abstract}

\section{INTRODUCTION}

Aircraft crew members are exposed to cosmic radiation of galactic and solar origin as well as to secondary radiation produced in the atmosphere and by interactions with the aircraft structure and its contents. Following recommendations of the International Commission on Radiological Protection (ICRP) in Publication $60^{(1)}$, the European Union (EU) introduced a revised Basic Safety Standards Directive ${ }^{(2)}$ which included exposure to natural sources of ionizing radiation, including cosmic radiation, as occupational exposure. Several European research projects have been focused on measurements on-board aircraft and modelling of the radiation exposure in flight altitudes with great success ${ }^{(3-8)}$. Due to an initiative by EURADOS, a working group was established (Working Group 5 on Aircraft Crew Dosimetry) to co-ordinate research, including collating and summarizing the scientific results and practical consequences of the measurements performed at workplaces of

\footnotetext{
${ }^{\dagger}$ Deceased.

*Corresponding author: peter.beck@arcs.ac.at
}

aircraft crew at flight altitudes. Initiated partly by the same group of scientists an ISO standard was proposed. Part 1 of the standard has already been finalised and is published ${ }^{(9)}$. Measurements and calculations of the radiation exposure due to solar events were not included in the EURADOS working group report and the ISO standard on cosmic radiation exposure on aircraft crew. Therefore, as an initiative of EURADOS, a co-ordinated research project was started on modelling of the radiation exposure due to solar particle events and validation of the models. In addition, the effects of short-time decreases in cosmic radiation intensity (Forbush decreases) in association with solar storms are to be examined. This project is part of work package 6 of the European research project CONRAD (Coordinated Network for Radiation Dosimetry). Work package 6 is concerned with the co-ordination of research in EU member states on the evaluation of complex mixed radiation fields at workplaces and is organised in two taskgroups. One task-group consisting of 14 members from 12 different European research institutes is working on aircraft crew radiation workplaces, with the objective of co-ordinating research activities in 
model improvements for radiation dose assessment due to solar particle events. The results aid European research, increase the efficiency of resource utilisation, facilitate the technology transfer to practical application and support the development of standards. The work was organised by means of meetings to exchange information on research activities and co-ordinate progress. The co-ordination work was carried out in close co-operation with the task-group on complex fields at workplaces at high energy particle accelerators and in the nuclear industry. There was close liaison between both task-groups with joint meetings and benchmark measurements. The task-group on solar energetic particle events provided validation of models for dose assessment of solar particle events, using data from neutron ground level monitors, in-flight measurement results obtained during a solar particle event and proton data measured by instruments on board satellite. This article summarises the validation study of improved methods for the characterisation and dosimetry of the exposure of aircraft crew from solar particle events. In the following, an outline is given of onboard measurements as well as predictions of the radiation exposure during solar particle events leading to a so-called ground level enhancement (GLE).

\section{RADIATION MEASUREMENTS ON-BOARD}

The exposure on board aircraft due to galactic cosmic radiation was extensively measured during the last decade ${ }^{(2)}$. Some of the research institutes installed radiation monitors on board aircraft for long-term investigations ${ }^{(4,9)}$. These institutes were able to gather data during some solar particle events and extended solar storms. For monitoring of radiation exposure due to solar events, active radiation instruments were used such as tissue equivalent proportional counter (TEPC), Geiger-Müller counter or Si-semiconductor detectors or spectrometers. Figures 1 and 2 show in-flight measurement results during a solar event on 15 April 2001 which lead to the ground level event GLE60 ${ }^{(10-12)}$. Both measurements show a significant increase of the radiation exposure during the flight for a time period of up to $3 \mathrm{~h}$. The total increase in terms of the radiation quantity $H^{*}(10)$ was about $50 \%$.

Figure 3 summarises the whole scenario of radiation exposure, including proton fluence rates measured by satellite, and ground level neutron monitor count rates during a solar storm which happened between October and November 2003 (Halloween storms) $^{(21)}$. The radiation monitor used was a TEPC showing the low- and high-LET contribution separately, installed on-board a Lufthansa Airbus A340. The ratio of low- and high-LET components is significantly different between GLE65 on 28 October 2003 and the following Forbush decrease.

Figure 4 shows the relative deviation of the radiation exposure expressed in terms of $H^{*}(10)$ during the solar storm period associated with GLE65 and the subsequent Forbush decrease compared with quiet periods before and after the storm. While the variation of radiation exposure for the same flight routes is about $\pm 10 \%$ in $12 \mathrm{~km}$ flight altitude, the increase during the Halloween storm was about $40 \%$ due to GLE65. On the other hand, the Forbush decrease that started on the 29 October 2003 (Figure 3) led 30\% decrease of the exposure on board aircraft. The mean quality factor of the radiation field for flights during normal cosmic radiation conditions shows $\mathrm{Q}=2.2$, during GLE $65 \mathrm{Q}=2.5$ and during Forbush decrease $\mathrm{Q}=2.1$. The uncertainty bars indicate a measurement uncertainty of $10 \%$ (one standard deviation) ${ }^{(8)}$. Measurements performed with the Liulin equipment on board a Czech Airlines aircraft during the flight Sofia to Prague just

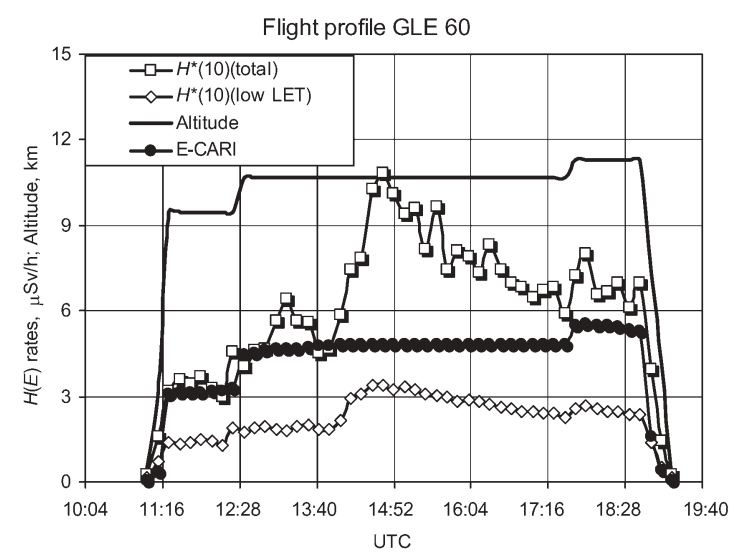

Figure 1. LIULIN measurements of GLE 60 during a flight from Prague to New York ${ }^{(12)}$. The calculation using the code CARI-6 does not include the radiation exposure of the GLE. 
VALIDATION OF MODELLING THE RADIATION EXPOSURE

ACREM measurements during GLE 60, 15 April 2001

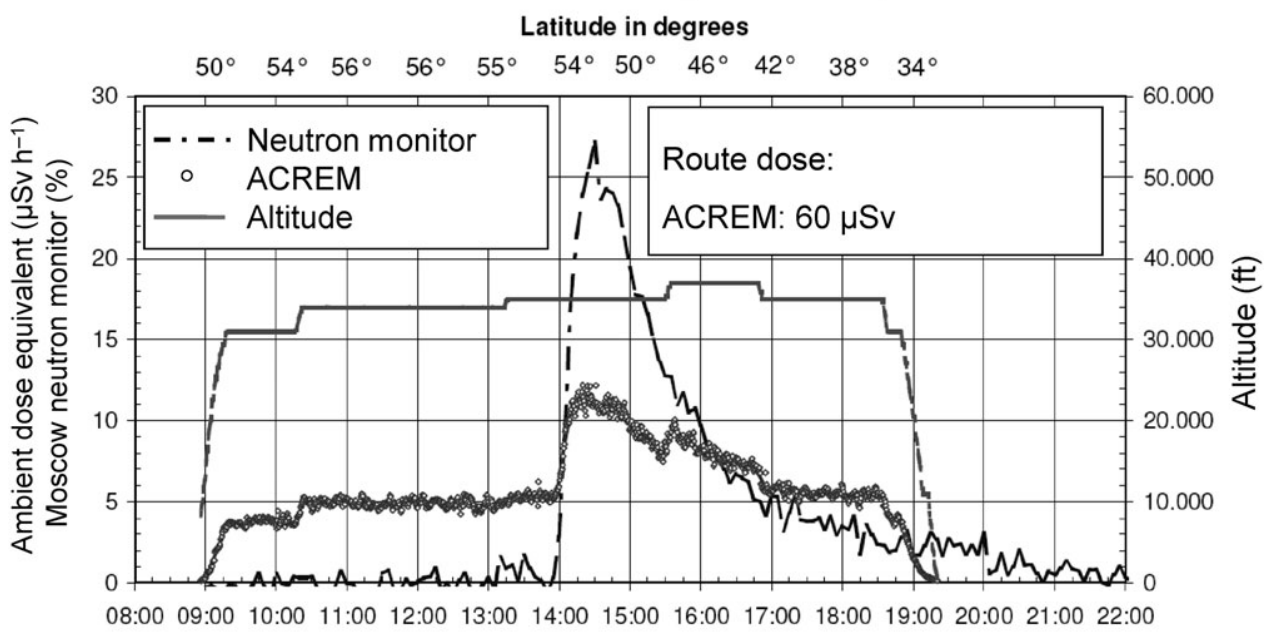

Flight Time (UTC)

Figure 2. ACREM in-flight measurement of GLE 60 during flight from Frankfurt to Dallas Fort Worth ${ }^{(11)}$, compared with neutron monitor data from the ground station at Moscow ${ }^{(22)}$. ACREM route dose in $H^{*}(10)=60 \mu$ Sv. AVIDOS ${ }^{(23)}$ calculates $H^{*}(10)=39 \mu \mathrm{Sv}(E=46 \mu \mathrm{Sv})$ and $\operatorname{EPCARD}^{(13)} H^{*}(10)=42 \mu \mathrm{Sv}(E=50 \mu \mathrm{Sv})$. The calculation by the codes does not include the radiation exposure of the GLE.
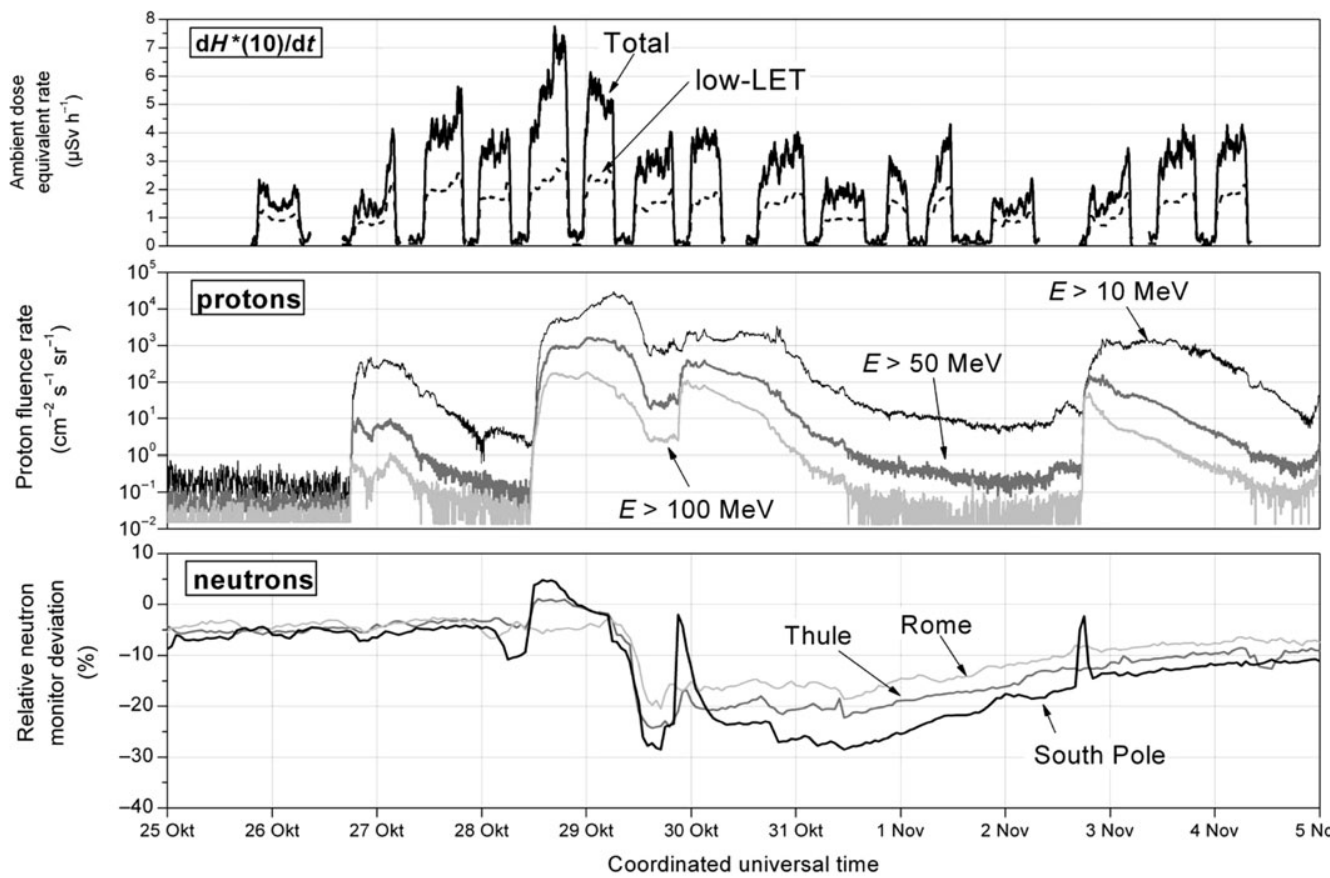

Figure 3. TEPC measurements in terms of $H^{*}(10)$ during the Halloween storms between October and November 2001 (above). The total dose and the low-LET contribution are shown. Proton fluence rate measured by NOAA satellite GEOS (middle). Neutron monitor count rates related to normal conditions before the solar storm in percent from the monitors at Thule, at Rome and at the South Pole (below). GLE65, GLE66 and GLE67 occurred during these time period ${ }^{(21)}$. 


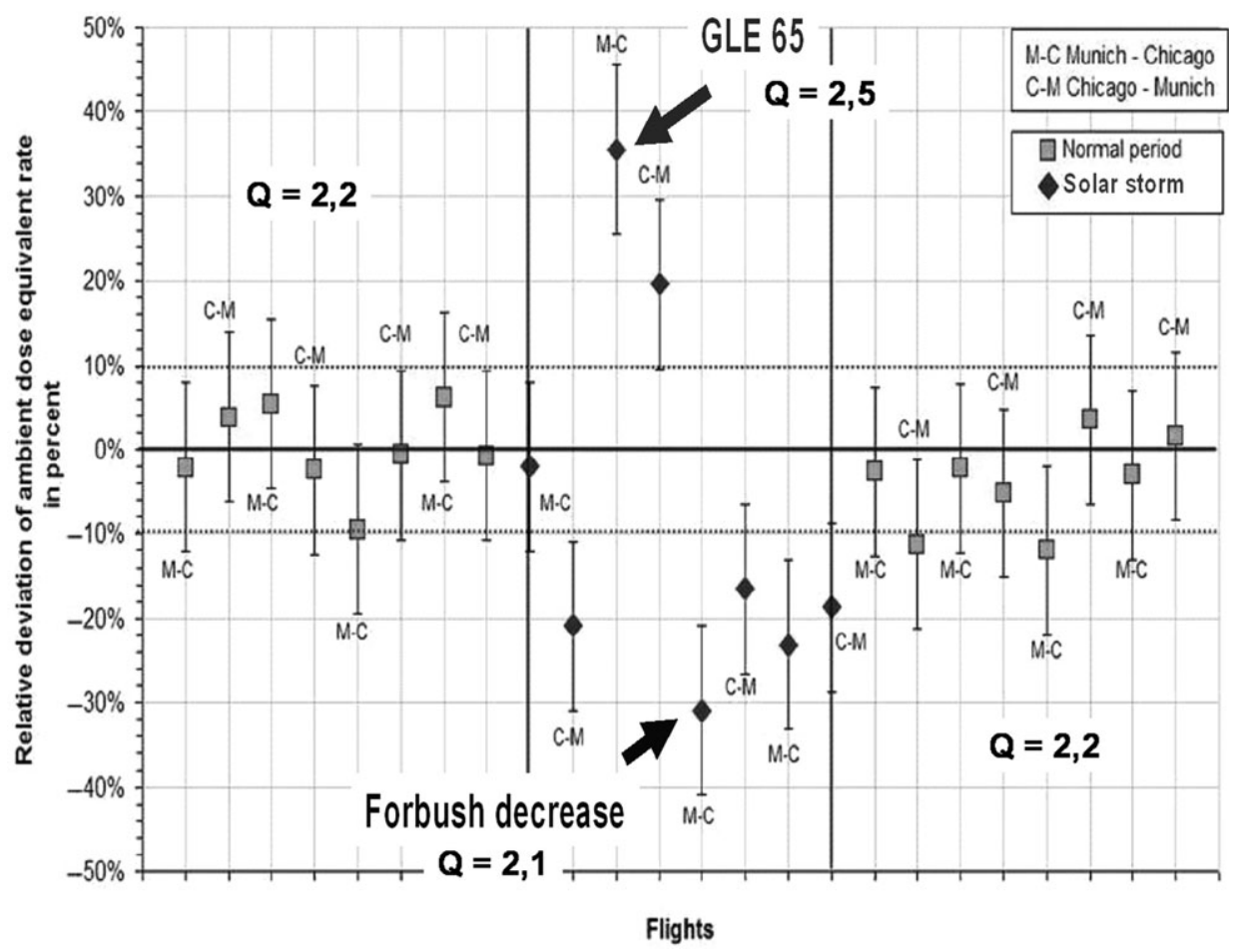

Figure 4. Observation of about $40 \%$ increase and 30\% decrease of the radiation exposure in $12 \mathrm{~km}$ due to GLE 65 and the following Forbush decrease ${ }^{(21)}$ compared to the normal cosmic radiation condition before the solar storm. The mean quality factor of the radiation field for flights during normal cosmic radiation conditions is 2.2 , during GLE $65 \mathrm{Q}=2.5$, and during

Forbush decrease $\mathrm{Q}=2.1$. The uncertainty bars indicate a measurement uncertainty of $10 \%$ (one standard deviation).

in the deep decrease showed that the exposure was about $28 \%$ lower when compared to normal solar activity conditions ${ }^{(12)}$. Further observations were reported earlier ${ }^{(14)}$.

\section{SOLAR EVENT CALCULATION}

The radiation exposure due to solar particle events leading to a GLE were estimated by some members of the task-group based on models, neutron ground level monitor data, proton data measured by satellites and in-flight data measured on board aircraft during GLEs (PLANETOCOSMICS ${ }^{(15)}$, SiGLE $^{(16)}$ and QARM $\left.^{(17)}\right)$. Several institutes of the task-group are working on complex calculation methods based on SPE proton input spectra, atmospherics and geomagnetic models, and using high energy transport Monte Carlo codes (e.g. FLUKA, GEANT4 and MCNPX).

Figure 5 shows the radiation exposure estimations for flights between Paris and New York with Concorde aircraft and between Paris and San Francisco for sub-sonic flights during 31 GLEs $(1942-2001)^{(18)}$. Total doses up to $\sim 6 \mathrm{mSv}$ per flight were calculated. The doses presented here correspond to the worst case scenario in terms of time departure of the flights, i.e. a time departure was selected leading to the maximum route dose. This provides an upper limit for the radiation dose. Most of the GLE contributions to the total route dose are lower than $100 \mu \mathrm{Sv}$ per flight.

Figure 6 shows a map of the peak neutron fluence rate $>10 \mathrm{MeV}$ in neutron per second per $\mathrm{cm}^{2}$ for the 29 September 1989 event and is calculated for $12 \mathrm{~km}$ altitude using the QARM model. It is typical for GLE's that the highest dose is observed around the Poles with almost no effect around the equator. For anisotropic events, the situation may be different. The QARM model uses ground level neutron monitor data plus GOES spacecraft data and has been validated against Concorde data for this event as well as the events of October $1989^{(19)}$. More recently, comparison has been made with measurements of the 15 April 2001 event as shown in Figure $7^{(20)}$.

Figure 8 shows world dose maps calculated for the radiation conditions during GLE69 on 20 January 2005 based on the PLANETOCOSMICS ${ }^{(15)}$ and SiGLE model ${ }^{(16)}$. Both calculation codes show the effective dose distribution between 7:04 and 7:16 UTC in a standard barometric altitude of $40000 \mathrm{ft}$. 


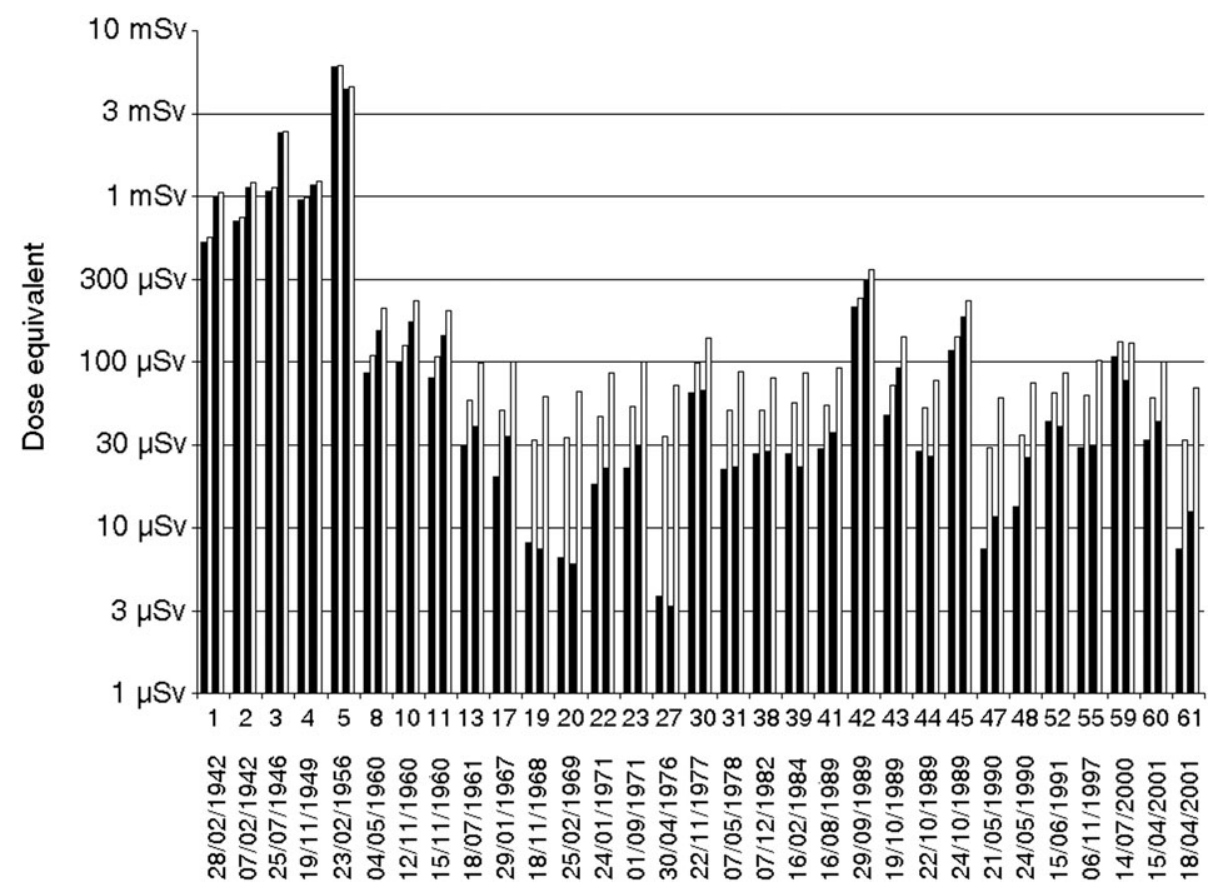

Figure 5. Worst case calculation performed using $\mathrm{SiGLE}^{(16)}$ for 31 GLEs for flights between Paris and New York with Concorde (first and second bars) and Paris to San Francisco with subsonic aircraft (third and fourth bars). GLE contribution (black bar), total dose (white bar). The GLEs taken into account are those significant in terms of radiation dose $e^{(18)}$.

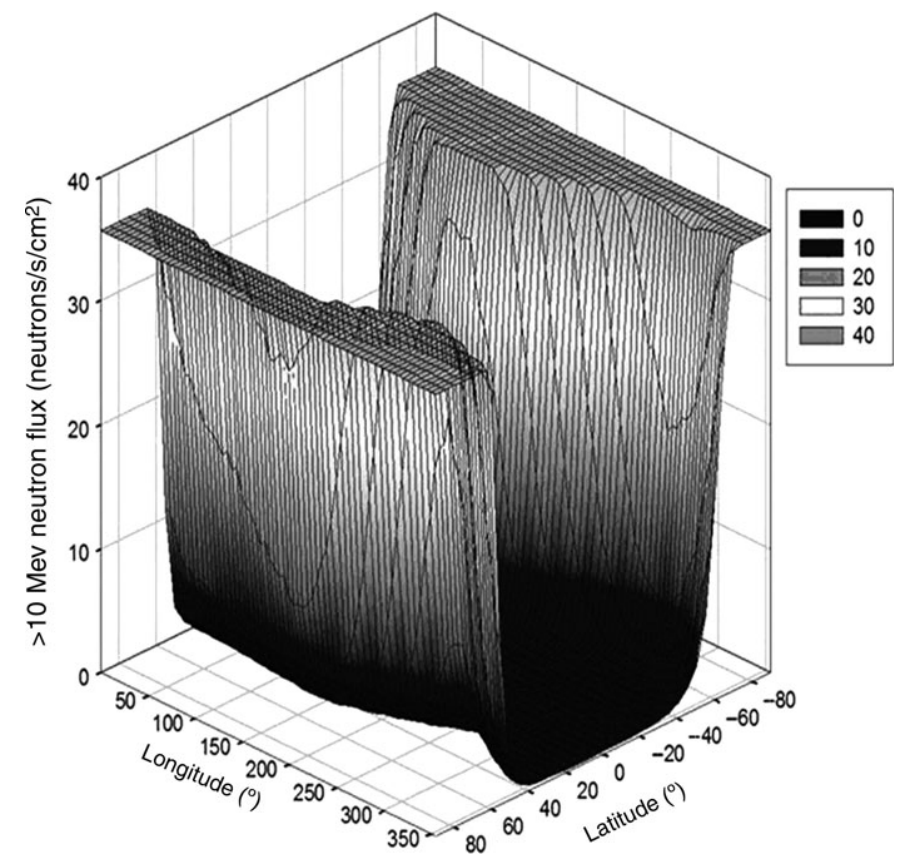

Figure 6. Neutron fluence rate for neutron with energy larger than $10 \mathrm{MeV}$ during maximum of GLE 42, 29 September $1989,12 \mathrm{~km}$ altitude. 

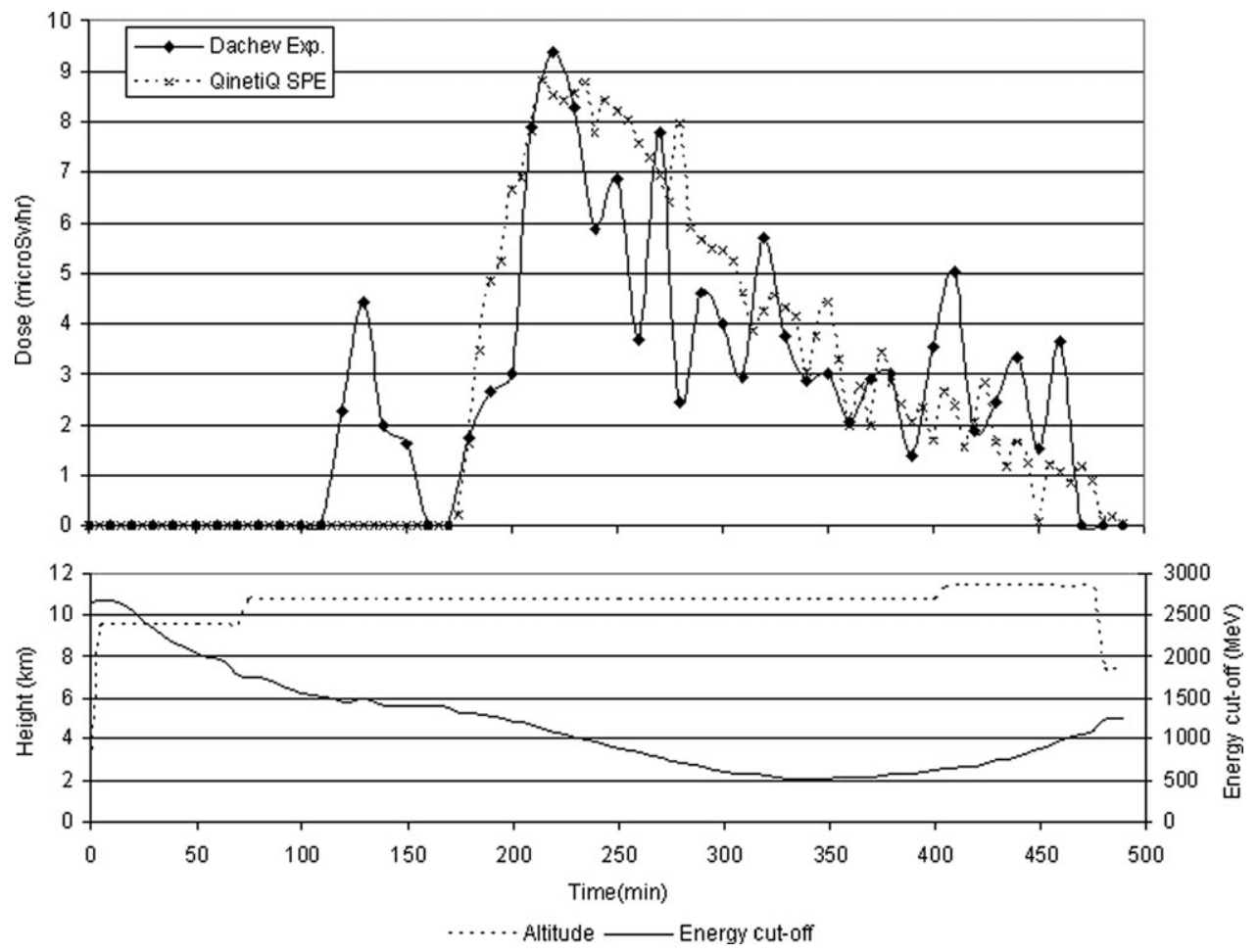

Figure 7. Comparison of on-board measurements of solar particle dose rates with GCR contribution subtracted (see Figure 1) with QARM calculations. Flight from Prague to New York during GLE 60, 15 April 2001 ${ }^{(19)}$.

In some parts, the agreement is reasonably well; in the area of the peak differences up to one order of magnitude were analysed. The current version of SiGLE does not take into account the anisotropy of a GLE. This can lead to significant deviations compared with calculations of the model PLANETOCOSMICS for highly anisotropic GLE's. Further differences are also due to the model constructions themselves.

\section{CONCLUSION}

A EURADOS consortium of 12 institutes coordinates research on radiation effects of solar energetic particles at aircraft altitudes. A comparison of different models and on-board measurements during selected GLE's are carried out. Calculated $H^{*}(10)$ values of the radiation exposure due to GLEs range up to a total dose of some $5 \mathrm{mSv}$ per route on board supersonic flights and up to some $1 \mathrm{mSv}$ on board subsonic flights crossing the Polar region. It has been observed that the radiation quality factor $Q$ during a GLE is significantly larger compared to normal cosmic radiation. The significant dose increase due to a GLE is most likely around the Poles, but not in the Equator region. The occurrence of GLEs is on average one event per year giving a route dose a few tens of $\mu \mathrm{Sv}$, one event per decade giving a route dose of a few hundred $\mu \mathrm{Sv}$ and perhaps one event per century giving a route dose of the order of mSv. GLEs are most probable during the solar maximum, with an increased likelihood before and after the maximum of sun's activity of the 11 year solar cycle. Several in flight measurements have been performed during GLEs. Calculations of radiation exposure due to GLEs are shown. Some models agree with limited experimental data reasonably well. Analysis of GLEs during geomagnetically disturbed conditions is still complex and time-consuming. Currently available solar particle event models can disagree with each other by an order of one magnitude. Further research is needed to continue the development of calculation models. Further, on-board measurements are required for the verification of SPE calculation models. On-board measurements are particularly important during the upcoming years of the increasing solar activity during solar cycle 24 . 

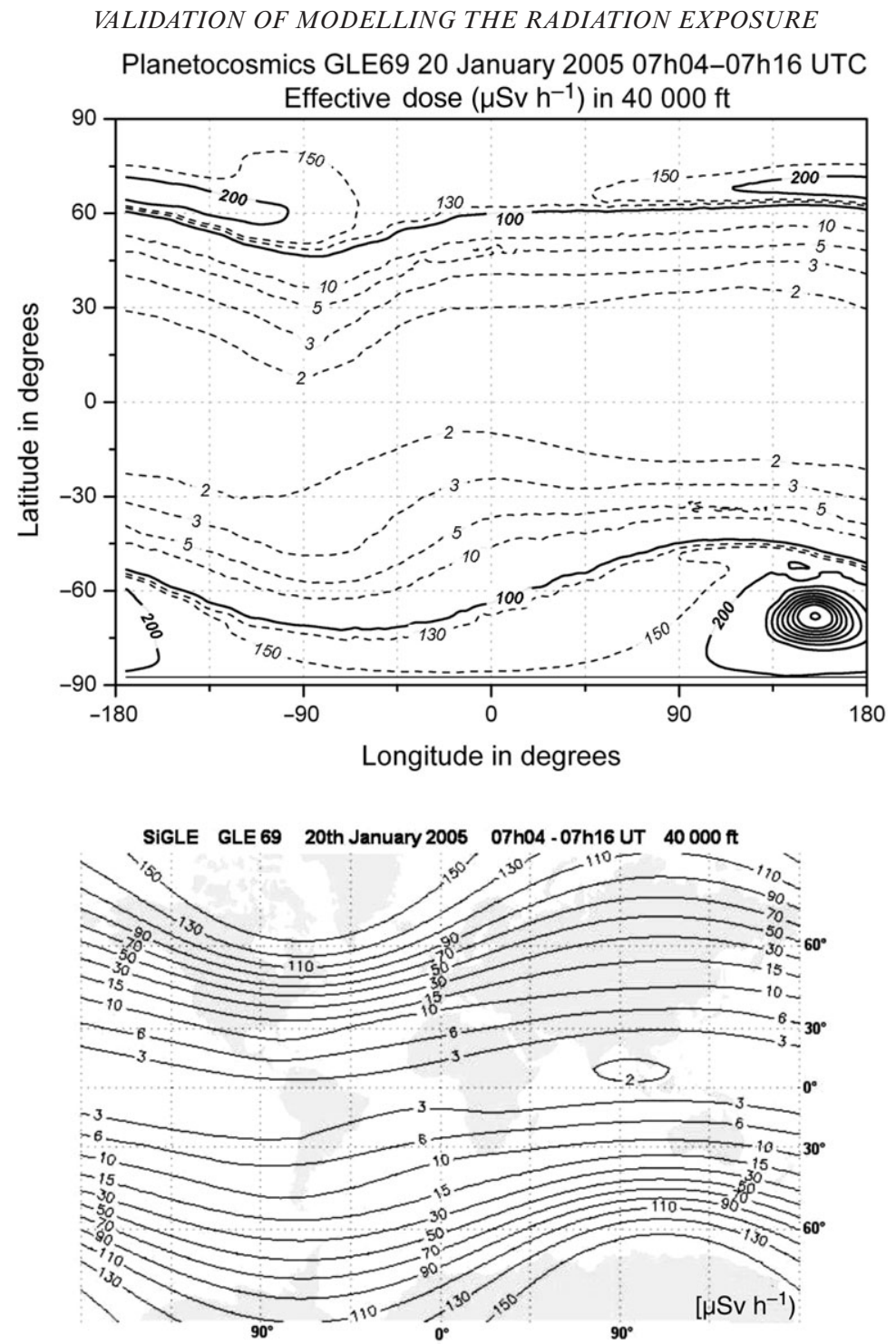

Figure 8. World dose map in terms of effective dose rate $\left(\mu \mathrm{Sv} \mathrm{h}{ }^{-1}\right)$, calculated for the conditions during the maximum of GLE 69 on 20 January 2005, 7:04 to 7:16 UTC, based on the models PLANETOCOSMICS (above) ${ }^{(15)}$ and SiGLE (below) ${ }^{(16)}$. Data are given for the total dose (GLE and the galactic cosmic radiation).

\section{FUNDING}

This work was financially supported by the European Commission, contract number FP6-12684. Further results of research part-funded by the European Commission, Directorate-General Research, under the auspices of the European Commission RTD Programme: Nuclear Energy (Euratom Framework Programme V, 1998-2002) under Contract No. FIGM-CT-2000-00068, national research funding, and research supported by Austrian Research Centers (Austria), Health Protection Agency (UK), Institute of Nuclear Physics (Poland), QinetiQ, Space Department (UK), University of Berne, (Switzerland), Paris-Meudon Observatoire (France), German Aerospace Center, Radiation Biology Department (Germany), HMGU, Institute of Radiation Protection (Germany), Czech Academy of Science, Nuclear Physics Institute (Czech Republic), National Physical 


\section{P. BECK ET AL.}

Laboratory (UK), Institute de Radioprotection et de Sûreté Nucléaire (France), Physikalisch-Technische Bundesanstalt, Braunschweig (Germany).

\section{ACKNOWLEDGEMENTS}

The CONRAD WP6/SG-B members and contributors to the study thank Aer Lingus, Air Canada, Air Emirates, Air France, Air New Zealand, Alitalia, Austrian Airlines, British Airways, Czech Airlines, Finnair, Iberia, Icelandair, Lufthansa, NASA, Scandinavian Airlines System, VARIG, and Virgin Atlantic Airways, for their assistance during in-flight measurements.

\section{REFERENCES}

1. International Commission on Radiological Protection. 1990 recommendations of the International Commission on Radiological Protection. ICRP Publication 60. Ann. ICRP 21(1-3) (Oxford: Pergamon Press) (1991).

2. Commission of the European Communities. Council Directive 96/29 EURATOM of 13 May 1996 laying down basic safety standards for the protection of the health of workers and the general public against dangers arising from ionizing radiation. Off. J. Eur. Commun. L159, 39 (1996).

3. Lindborg, L., Bartlett, T. D., Beck, P., McAulay, I. R., Schraube, H., Schnuer, K. and Spurný, F. (eds). Cosmic Radiation Exposure of Aircraft Crew: Compilation of Measured and Calculated data. EC Report Radiation Protection 140, DG TREN (Luxemburg ) pp. 1-271 (2004) (ISBN 92-894-8448-9).

4. Beck, P., Ambrosi, P., Schrewe, U. and O'Brien, K. ACREM, Aircrew Radiation Exposure Monitoring. ARC Seibersdorf research. Report OEFZS-G-0008 (1999).

5. Dublin Institute for Advanced Studies. Dosimetry of Aircrew Exposure to Radiation Solar Maximum. Dublin, Dublin Institute for Advanced Studies (DIAS), FIGM-CT-2000-00068, 29 (2001).

6. Regula, D. and David, J. Measurements of cosmic radiation on board Lufthansa aircraft on the major intercontinental flight routes. Radiat. Prot. Dosim. 48(1), 651-672 (1993)

7. Regulla, D. and Schraube, H. Radiation exposure of aircrews in civil aviation. Strahlenbiologie und Strahlenschutz. 28. Jahrestagung des Fachverbands für Strahlenschutz e.V., Hannover, 23.-25.10.1996 (Hrsg.: G. Heinemann, H. Pfob). Köln, Verlag TÜV Rheinland, pp. 375-380 (1996).

8. Lillhök, J.A. et al. A comparison of ambient dose equivalent meters and dose calculations at constant flight conditions. Radiat. Meas. 42(3), 323-333 (2007).

9. International Organization for Standardization. Dosimetry for exposures to cosmic radiation in civilian
aircraft-Part 1: Conceptual basis for measurements. ISO ISO 20785-1 (2006).

10. Spurny, F. and Dachev, T. Long-term monitoring of the onboard aircraft exposure level with Si-diode based spectrometer. Adv. Space Res. 32, 53-58 (2003).

11. Bartlett, D. T. et al. Investigation of Radiation Doses at Aircraft Altitudes during a Complete Solar Cycle. European Space Agency, (Special Publication) ESA SP (477), pp. 525-528 (2002).

12. Spurný, F. and Dachev, Ts. Measurements in an Aircraft during an Intense Solar Flare, Ground Level Event 60, on the 15th April 2001. Radiat. Prot. Dosim. 95, 273-275 (2001).

13. Schraube, H., Leuthold, G., Heinrich, W., Roesler, S., Mares, V. and Schraube, G. EPCARD-European Program Package fort he Calculation of Aviation Route Doses. GSF-Report 08/02, Neuherberg (2002). Available on http://helmholtz-muenchen.de/epcard).

14. Kyllönen, J.-E., Lindborg, L. and Samuelson, G. Cosmic radiation measurements on-board aircraft with the variance method. Radiat. Prot. Dosim. 93, 197-205 (2001).

15. Bütikofer, R., Flückiger, E. O., Desorgher, L. and Moser, M. R. The extreme solar cosmic ray particle event on January 2005 and its influence on the radiation dose rate at aircraft altitude. Sci. Total Environ. 391, 177-183 (2008).

16. Lantos, P. Radiation doses potentially received on-board aeroplane during recent solar particle events. Radiat. Prot. Dosim. 118, 363-374 (2006).

17. Lei, F., Clucas, S., Dyer, C. and Truscott, P. An atmospheric radiation model based on response matrices generated by detailed Monte Carlo simulations of cosmic ray interactions. IEEE Trans. Nucl. Sci. 51(6), 3442-3451 (2004).

18. Lantos, P. and Fuller, N. History of the solar flare radiation doses on-board aeroplanes using semi-empirical model and Concorde measurements. Radiat. Prot. Dosim. 104(3), 199-210 (2003).

19. Dyer, C. S., Lei, F., Clucas, S. N., Smart, D. F. and Shea, M. A. Calculations and observations of solar particle enhancements to the radiation environment at aircraft altitudes. Adv. Space Res. 32, 81-93 (2003).

20. Dyer, C., Lei, F., Hands, A. and Truscott, P. Solar particle events in the QinetiQ atmospheric radiation model. IEEE. Trans. Nucl. Sci. 54(4), 1071-1075 (2007).

21. Beck, P., Latocha, M., Rollet, S. and Stehno, G. TEPC reference measurements at aircraft altitudes during a solar storm. Adv. Space Res. 36(9), 1627-1633 (2005).

22. Real time neutron ground monitor station at Troitsk (Moscow). Available on http://helios.izmiran.troitsk. $\mathrm{ru} /$ cosray/main.htm (2001).

23. Beck, P., Latocha, M., Dorman, L., Pelliccioni, M. and Rollet, S. Measurements and simulations of the radiation exposure to aircraft crew workplaces due to cosmic radiation in the atmosphere. Radiat. Prot. Dosim. 126, 564-567 (2007). Available on http://radiation-seibersdorf.at/avidos. 